\title{
Our gratitude and congratulations to our guest editor Julian Eaton-Rye and thanks to all the 167 contributors to the special issue honoring professor Govindjee
}

\author{
GYÖZÖ GARAB
}

Biological Research Center, Hungarian Academy of Sciences, Szeged, Hungary and Department of Physics, University of Ostrava, Ostrava, Czech Republic

E-mail: garab.gyozo@brc.mta.hu

For the Special Issue in Photosynthetica, volume 56 (1): 1477 (2018) our heartfelt thanks go to Julian J. Eaton-Rye, who single-handedly edited this large issue with 45 papers, with 167 authors (see below for the alphabetical list) from 20 countries around the world: Australia, Azerbaijan, Canada, China, Czech Republic, Finland, Germany, Hungary, India, Japan, Mexico, Netherlands. New Zealand, Norway, Russia, Sweden, Taiwan, Thailand, UK and the USA. I had initiated the idea of this special issue for Govindjee's $85^{\text {th }}$ birthday; now, I want to say special thanks to Julian - it is the best Special Issue in Photosynthetica that I have ever seen; thanks to all the authors honoring Govindjee, and Julian (and a very large number of reviewers) for making this happen in such an excellent way! I take this opportunity to say thanks to Helena Synková and Ivana Stětinová for their continued support. For earlier honors to Govindjee at his $75^{\text {th }}$ birthday, see Eaton-Rye (2007a). Eaton-Rye (2007b) and Jajoo et al. (2009); and at his $80^{\text {th }}$ birthday, see Allakhverdiev et al.
(2013), Eaton-Rye (2012, 2013), Eaton-Rye et al. (2012) and Prášil (2014).

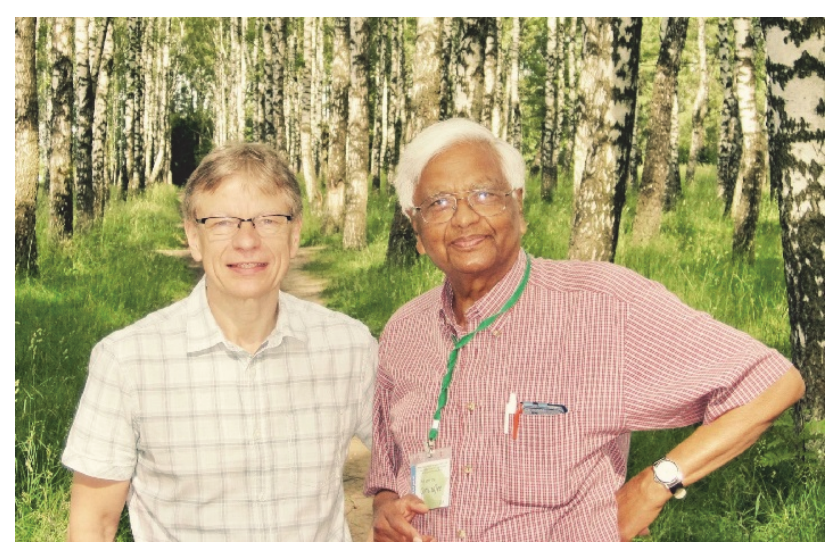

Fig. 1. Julian J. Eaton-Rye (left) and Govindjee (right) in 2016 in Pushchino, Russia. Photo by G. Garab.

We thank the following 167 authors (with their full names) for their wonderful contributions to remember our dear friend Govindjee, known as Mr. Photosynthesis, who now hopes to be known as Govindjee Govindjee.

Alonsa M. Acuna

William W. Adams

Parveen Akhtar

Suleyman I. Allakhverdiev

Gennady Ananyev

Yusuke Arai

Eva-Mari Aro

Anindita Bandyopadhyay

Jesús Barrera-Rojas

Anna M. Beiler

Basanti Biswal

Sandeo Biswas

Karen Bitan

Lars Olof Björn

Alex Yu. Borisov

Barry D. Bruce

Danny P. Brummel

Tanai Cardona

Jeannine Cavender-Bares
Steven Hui-Yuan Chen
Min Chen
Yi-Fang Chiu
Wah Soon (Fred) Chow
Hsiu-An Chu
Christopher M. Cohu
Barbara Demmig-Adams
G. Charles (Chuck) Dismukes
Julian J. Eaton-Rye
Paul G. Falkowski
Marco Flores
John A. Gamon
Fudan Gao
Győző Garab
Colin Gates
Zoltán Gombos
Carlos Gomez-Lozero

Jeannine Cavender-Bares

Min Chen

Yi-Fang Chiu

Hsiu-An Chu

Christopher M. Cohu

Barbara Demmig-Adams

G. Charles (Chuck) Dismukes

Paul G. Falkowski

Marco Flores

A. Gamon

Győző Garab

Carlos Gomez-Lozero
Govindjee Govindjee

Emma Berta Gutierrez-Cirlos

Guangye Han

Volker Hartmann

Brian Haschemeyer

Éva Herman

Priscila Herrera-Salgado

Martin F. Hohmann-Marriott

Jine-Yung Huang

Xuan-Dong Huang

Nien-Tzu Hung

Mizuho Ichinose

Aran Incharoensakdi

Masako Iwai

Lakshmi Jain

Anjana Jajoo

Rohit Joshi

Radek Kaňa 
Andrew A. Khorobrykh

Alexandra Yu. Khudyakova

Diana Khusnutdinova

Mariann Kis

Jana Knoppova

Vyacheslav (Slava) V. Klimov ${ }^{\dagger}$

Koichi Kobayashi

Zbigniew S. Kolber

Josef Komenda

Junpei Kondo

Christos Kotakis

Shan Kothari

László Kovács

Elmars Krausz

Vladimir D. Kreslavski

Johannes (Wanne) Kromdijk

Hajnalka Laczkó-Dobos

Jake J. Lamb

Petar H. Lambrev

Julian Langley

Anthony (Tony) W.D. Larkum

Allison Lauria

Dušan Lazár

Lourdes Elizabeth Leyva-Castillo

Mei Li

Yaqiong Li

Keng-Min Lin

Lucas Loveless

Yan Lu

Valery Yu. Lyubimov

Weimin Ma

Pirkko Maenppa

Akari Makita

Mahir Mamedov

Péter Maróti

Peter Mathur

Michael (Mike) S. McBride

Johannes Messinger
Jyotirmoy Mondal

Rebecca A. Montgomery

Gary F. Moore

Jennifer Morton

Ryo Nagao

Kärin Nickelsen

Peter J. Nixon

Takumi Noguchi

Arthur M. Nonomura

Ekaterina S. Nosikova

Marc M. Nowaczyk

Teruo Ogawa

Ai Ohnishi

Hisataka Ohta

Himadri B. Pakrasi

Jitendra Kumar Pandey

Ashwani Pareek

Uday Vasudev Pathre

Andrew Pedersen

Chang-Lian Peng

Stephanie K. Polutchko

Ondřej Prášil

Robin Purchase

Wuttinun Raksajit

John A. Raven

Emmanuel Rios-Castro

Raymond James Ritchie

Matthias Rögner

Gunvor Rokke

Adrian Ruff

Prafullachandra Vishnu (Raj) Sane

Wolfgang Schuhmann

Alexey Yu. Semenov

Galina A. Semenova

Shengxi Shao

Jian-Ren Shen

Lianglian Shen

Dmitriy (Dima) Shevela
Pramod Arvind Shirke

Galina N. Shirshikova

Alexander N. Shmarev

Sneh Lata Singla-Pareek

Gábor Sipka

James Leonard Smart

Jared J. Stewart

Alexandrina (Sandra) Stirbet

Adela Straskova

Mamoru Sugita

Lirong Tian

Konstantin G. Tikhonov

Tatsuya Tomo

Hiromichi Uchiyama

Sindhujaa Vajraval

Pascal van Alphen

Rienk van Grondelle

Ivo H.M. van Stokkum

Natalie Vella

Amy S. Verhoeven

Lia A. Vitukhnovskaya

Wim J. Vredenberg

Hazime Wada

Ran Wang

Silas Wungrampha

Masato Yamada

Denis Y. Yanykin

Panutda Yodsang

Zhen-Chao $\mathrm{Yu}$

Tomas Zakar

Andrey A. Zaspa

Ling-Da Zeng

Tai-Jie Zhang

Sergey K. Zharmukhamedov

Jin Zheng

Hui Zhu

Ottó Zsiros

Acknowledgement: I am grateful to professor Govindjee for his generous help in composing this list of authors.

\section{References}

Allahkhverdiev S.I., Shen J.-R., Edwards G.E.: Special issues on Photosynthesis Education honoring Govindjee. - Photosynth. Res. 116: 107-110, 2013.

Eaton-Rye J.J.: Celebrating Govindjee's 50 years in photosynthesis research and his 75 th birthday. - Photosynth. Res. 93: 1-5, 2007a.

Eaton-Rye J.J.: Snapshots of the Govindjee lab from the late 1960s to the late 1990s and beyond. - Photosynth. Res. 94: 153- 178, $2007 \mathrm{~b}$

Eaton-Rye J.J.: Contributions of Govindjee, 1970-1999. - In: Eaton-Rye J.J., Tripathy B.C., Sharkey T.D. (ed.): Photosynthesis: Plastid Biology, Energy Conversion and Carbon Assimilation. Advances in Photosynthesis and Respiration. Vol. 34. Pp. 815-834. Springer, Dordrecht 2012.

Eaton-Rye, J.J.: Govindjee at 80: more then 50 years of free energy for photosynthesis. - Photosynth. Res. 116: 111-144, 2013.

Eaton-Rye, J.J.: Foreword - Photosynthetica 56: 1-10, 2018.

Eaton-Rye J.J., Tripathy B.C., Sharkey T.D. (ed.): Photosynthesis: Plastid Biology, Energy Conversion and CarbonAssimilation. Advances in Photosynthesis and Respiration. Vol. 34. Pp. 854. Springer, Dordrecht 2012.

Jajoo A., Guruprasad K.N., Bharti S., Mohanty P.: International conference "Photosynthesis in the Global Perspective" held in honor of Govindjee, November 27-29, 2008, Indore, India. - Photosynth. Res. 100: 49-55, 2009.

Prášil O.: Govindjee, an institution, at his $80^{\text {th }}$ (really $81^{\text {st }}$ ) birthday in October 2013: a pictorial essay. - Photosynth. Res. 122: 113-119, 2014. 